\title{
Pan-drug-resistant and biofilm-producing strain of Burkholderia pseudomallei: first report of melioidosis from a diabetic patient in Yogyakarta, Indonesia
}

This article was published in the following Dove Press journal:

International Medical Case Reports Journal

\author{
Titik Nuryastuti' \\ Nusaibah Umaroh ${ }^{2}$ \\ Rizka Humardewayanti \\ Asdie $^{3}$ \\ Ika Puspita Sari ${ }^{4}$ \\ Ahmad Musthafa' \\ 'Department of Microbiology, Faculty \\ of Medicine, Public Health and \\ Nursing, Universitas Gadjah Mada, \\ Yogyakarta, Indonesia; ${ }^{2}$ Pharmacy \\ Installation of Dr. Kariadi General \\ Hospital, Semarang, Center of Java, \\ Indonesia; ${ }^{3}$ Department of Internal \\ Medicine, Faculty of Medicine, Public \\ Health and Nursing, Universitas \\ Gadjah Mada/Sardjito Hospital, \\ Yogyakarta, Indonesia; ${ }^{4}$ Department of \\ Pharmacology and Clinical Pharmacy, \\ Faculty of Pharmacy, Universitas \\ Gadjah Mada, Yogyakarta, Indonesia
}

Correspondence: Titik Nuryastuti Department of Microbiology, Faculty of Medicine, Public Health and Nursing, Universitas Gadjah Mada, Farmaco Street, Sekip, Yogyakarta 5528I, Indonesia

Tel +62 274580297

Email t.nuryastuti@ugm.ac.id

\begin{abstract}
Melioidosis, an infectious disease caused by Burkholderia pseudomallei, has recently gained importance as an emerging infectious disease in Indonesia. Reports of this infection in Indonesia are limited, although cases have been reported in Makassar, South Sulawesi. We report a case of cutaneous melioidosis caused by pan-drug-resistant, moderate biofilm-producer strain of $B$. pseudomallei in a diabetic patient. To the best of our knowledge, this is the first case of melioidosis caused by multidrug resistant and biofilm-former strain of $B$. pseudomalle $i$ being reported from Yogyakarta Province, Indonesia. The patient was successfully treated with abscess drainage and debridement, including total contact casting and no antibiotic treatment.
\end{abstract} Keywords: melioidosis, biofilm, pan-drug-resistance, Burkholderia pseudomallei

\section{Introduction}

Melioidosis, caused by Burkholderia pseudomallei, is an epidemic-prone disease of public health importance in South East Asia and Northern Australia. ${ }^{1}$ The disease is spread by inhalation, ingestion, and inoculation, and less commonly through personto-person, sexual, perinatal, vertical, and nosocomial routes. ${ }^{1,2}$ It has a fascinating pathogenesis, and an extremely diverse clinical manifestation. ${ }^{2}$ Skin and soft tissue infections are a common manifestation of melioidosis and may be the source of systemic infection, or vice versa. Melioidosis mostly occurs in patients with preexisting conditions such as type-II diabetes, chronic disease of the lung or kidneys, excessive alcohol consumption, viral infection, and use of immunosuppressants, particularly steroids. ${ }^{1,3}$

The presence of closely related Burkholderia species in clinical specimen poses a major challenge in identifying B. pseudomallei. Rarity of melioidosis beyond endemic cases makes this microorganism difficult to be recognized, particularly by laboratories lacking properly validated diagnostic assay. ${ }^{4}$

B. pseudomallei naturally resists a large array of antimicrobial agents, such as macrolides, narrow-spectrum cephalosporins, most penicillins, polymyxins, and aminoglycosides. Moreover, clinical evidence indicates that fluoroquinolones are also ineffective. ${ }^{5,6}$

Bacterial biofilm poses further problems, because it provides the bacteria a means to withstand the host immune system and antimicrobial agents. Formation of biofilm, responsible for dormancy, latency, and relapse of melioidosis, likely allows persistent infection and a greater chance of asymptomatic infection. ${ }^{7,8}$ 
We report a case of melioidosis presenting as skin and soft tissue infection, caused by a pan-drug-resistant and biofilmproducer strain of B. pseudomallei in Yogyakarta, Indonesia.

\section{Case report}

We report a case of a 57-year-old man admitted to Dr Sardjito General Hospital. He complained of recurrent wound infection on his right sole (Figure 1). He was diagnosed with type-II diabetes 22 years ago and was treated with NovoMix ${ }^{\circledR}$ 30 (Novo Nordisk Indonesia LLC, Jakarta, Indonesia) thrice daily for the last 3 months. His ulcer started spontaneously as a blister 3 months ago. He had a history of similar ulcers on his right foot 7 years earlier. He has a history of hypertension and peripheral artery disease, but none of the following: coronary heart disease, stroke, deep vein thrombosis, or complications of the eye and kidneys. His vital signs were unremarkable. Physical examination on admission revealed an infected ulcer on the sole, $5 \times 4 \mathrm{~cm}$, with depth reaching the muscle layer. Both feet were dry and scaly, lightly hairy, hyperpigmented, and ulcerated. In addition, the right foot had thickened nails and Charcot foot. Laboratory examination found slight anemia ( $\mathrm{Hb} 11.5 \mathrm{~g} / \mathrm{dL}$ ), hyperglycemia (random blood glucose $337 \mathrm{mg} / \mathrm{dL}$ ), and low high-density lipoprotein $(9 \mathrm{mg} / \mathrm{dL})$. Feet and chest X-rays were unremarkable.

The patient refused to be hospitalized; therefore, microbiological examination could not be conducted. The patient was discharged with oral empiric antibiotics: cotrimoxazole 960 $\mathrm{mg}$ twice daily and clindamycin $300 \mathrm{mg}$ four times a day. His hypertension was treated with irbesartan $300 \mathrm{mg}$ once daily and his type-II diabetes was managed with Humalog (Eli Lilly Indonesia LLC [company presently closed], Jakarta, Indonesia) 16 units three times a day. Since the wound did not improve, culture and sensitivity tests were conducted, resulting in a scanty growth of Pseudomonas sp., resistant to gentamycin. His antibiotics were adjusted according to the sensitivity pattern. The patient was then discharged and given a 3 week course of oral cefixime and ciprofloxacin, with no improvement.

Since the patient had a history of recurrent abscesses, melioidosis was among the differential diagnoses. The culture and sensitivity tests were repeated. Microbiology examination on a swab sample from the wound base revealed $B$. pseudomallei. Culture on blood agar, MacConkey agar, and Ashdown agar yielded short Gram-negative rods with bipolar staining. On Ashdown agar, these colonies appeared pale pink, and after prolonged incubation became dark purple, dry, and wrinkled in the center (Figure 2). A very strong sweetish-putrid odor was noted, resembling stagnant water.
These characteristics are suggestive of B. pseudomallei. To confirm our finding, we conducted nested PCR to detect $16 \mathrm{~S}$ rRNA gene. We used an outer primer specific to the genus Burkholderia sp. and an inner primer specific to B. pseudomallei in the first and second amplification, respectively. Visualization of a $397 \mathrm{bp}$-sized DNA bands in electrophoresis with $1 \%$ agarose gel confirmed the presence of $16 S$ rRNA gene of B. pseudomallei (Figure 3). ${ }^{9}$

Antibiotic susceptibility test of $B$. pseudomallei was done in a biological safety cabinet, by disk diffusion method on

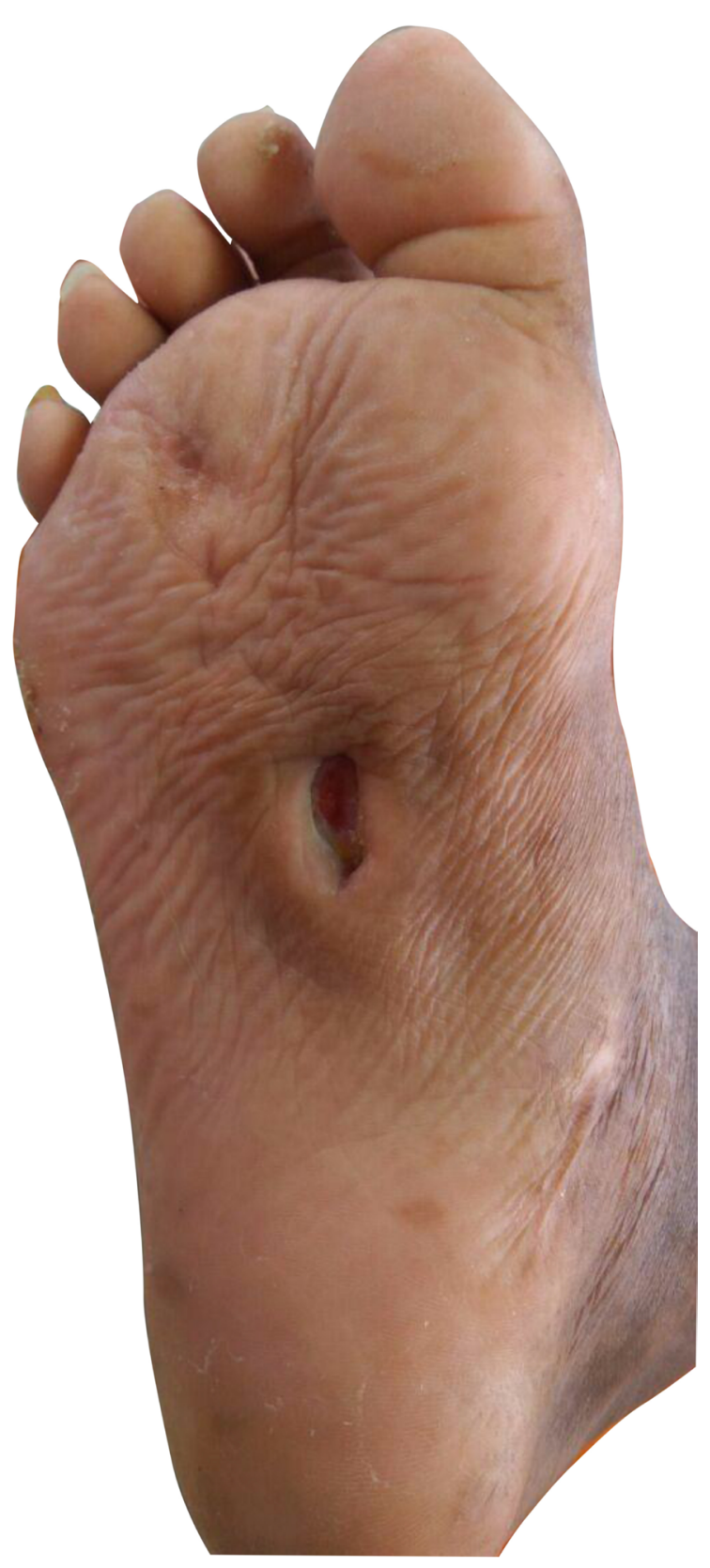

Figure I An ulcerated wound on the sole of right foot, measuring $5 \times 4 \mathrm{~cm}$. 
Mueller Hinton agar. The resulting zones of inhibition are demonstrated as uniformly circular with confluent lawns of growth. The Clinical and Laboratory Standards Institute has not published the interpretation result for $B$. pseudomallei. Therefore, we searched the guidelines and reference for the threshold of Burkholderia cepacia, Pseudomonas aeruginosa, or Enterobacteriaceae. ${ }^{10,11}$ The result revealed resistance to all antibiotics tested (Table 1).

We determined the $B$. pseudomallei biofilm-forming ability using a modified protocol. We standardized the bacterial density of the spectrophotometer $(\mathrm{OD} 600=1)$ prior to the test. Then we inoculated the overnight cultures of $B$. pseudomallei into brain heart infusion broth and incubated it aerobically overnight in a
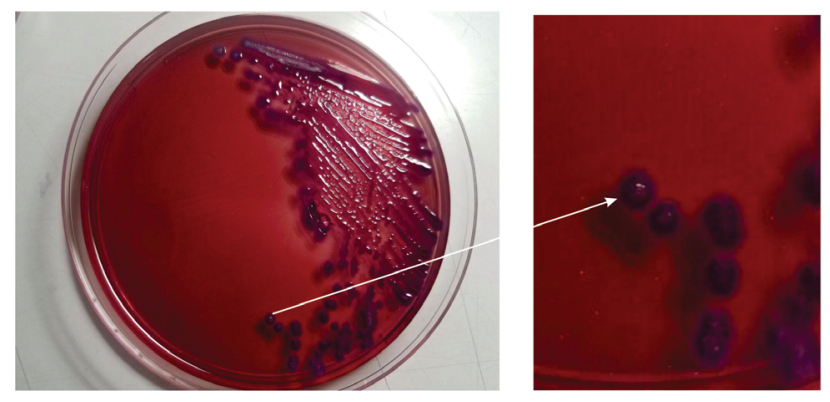

Figure 2 Morphology of B. pseudomallei colonies on ash down agar after 48 hours incubation at $37^{\circ} \mathrm{C}$.

Notes: The wrinkled surface of the colonies was appearing. The colony size ranges from 4 to $10 \mathrm{~mm}$.

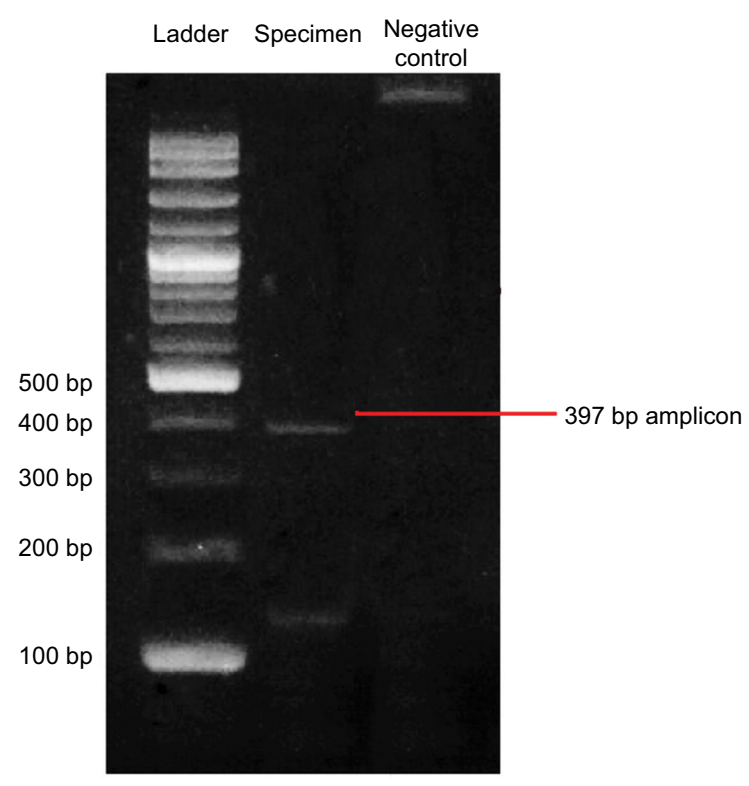

Figure 3 Result of PCR using a I6S rRNA primer.

Notes: Escherichia coli ATCC 35218 was used as negative control for this amplification assay. A product of 397 bp amplicon was present, which confirmed $B$. pseudomallei identification. shaking incubator at $37^{\circ} \mathrm{C}$. Afterward, we added $200 \mu \mathrm{L}$ bacterial suspensions to the wells of a 96-well flat bottom plastic tissue culture plate (Nunc, Roskilde, Denmark) and incubated it at $37^{\circ} \mathrm{C}$ for 48 hours. We used uninoculated Brain Heart Infusion broth as control. Following incubation, we washed the well three times to remove nonadherent bacteria. We then stained the wells with $50 \mu \mathrm{L}$ filtered $1 \%$ crystal violet for 5 minutes. We washed excess stain with water and air dried the wells. Next, we solubilized the crystal violet-bound bacterial cells with $200 \mu \mathrm{L}$ of $5 \%$ acid isopropanol and measured the released stain using a microplate reader at $595 \mathrm{~nm}$. We repeated this procedure eight times and took the average OD595. According to Hassan et al, ${ }^{12}$ the $B$. pseudomallei are considered a moderate biofilm-former strain as the OD595 was $0.75 \pm 0.03$.

The patient underwent surgical abscess-draining, debridement, and application of total contact casting to relieve body weight of the diabetic ulcer. He was followed up until September 2017. At the time of this writing, he was well without evidence of recurrence or relapse, and his ulcer had healed.

\section{Discussion}

Definitive diagnosis of melioidosis requires isolation and identification of $B$. pseudomallei from clinical specimens because its clinical manifestations vary widely. B. pseudomalle $i$ is classified as Biosafety Level 3 agent, necessitating the analyzing laboratory to be appropriately equipped. Little clinical experience and lack of pathognomonic symptoms hinder bacterial identification by laboratories faced with an unexpected case of melioidosis. Furthermore, identification of B. pseudomallei necessitates use of intensive microbiological workup, such as confirmatory amplification of $16 \mathrm{~S}$ rRNA gene.

Table I Antibiotic resistance pattern of B. pseudomallei performed by Kirby-Bauer method of disc diffusion assay

\begin{tabular}{|l|l|l|}
\hline $\begin{array}{l}\text { Class of } \\
\text { antibiotics }\end{array}$ & Antibiotics & $\begin{array}{l}\text { Resistance } \\
\text { pattern }\end{array}$ \\
\hline \multirow{2}{*}{ Penicillins } & Piperacillin & $\mathrm{R}$ \\
\cline { 2 - 3 } & Piperacillin-tazobactam & $\mathrm{R}$ \\
\hline \multirow{2}{*}{ Cephalosporins } & Ceftazidime & $\mathrm{R}$ \\
\cline { 2 - 3 } & Cefepime & $\mathrm{R}$ \\
\hline Carbapenems & Meropenem & $\mathrm{R}$ \\
\hline Monobactams & Aztreonam & $\mathrm{R}$ \\
\hline Aminoglycosides & Gentamycin & $\mathrm{R}$ \\
\cline { 2 - 3 } & Amikacin & $\mathrm{R}$ \\
\hline Fluoroquinolones & Ciprofloxacin & $\mathrm{R}$ \\
\cline { 2 - 3 } & Levofloxacin & $\mathrm{R}$ \\
\hline Sulfonamides & Cotrimoxazole & $\mathrm{R}$ \\
\hline Tetracyclines & Tetracycline & $\mathrm{R}$ \\
\hline
\end{tabular}

Note: R, resistant. 
The patient presented with mild, chronic, and localized skin and soft tissue infection. Based on these clinical manifestations, we considered it as primary cutaneous melioidosis. Skin and soft tissue infection, primary and secondary, account for $13 \%-24 \%$ of melioidosis in published case series. ${ }^{13,14}$ Primary skin melioidosis is often localized and less severe than other forms of melioidosis. On the other hand, primary skin melioidosis has been reported to be associated with necrotizing fasciitis, sepsis, and internal organ abscesses in Southeast Asia ${ }^{13,15}$

We deemed our case to be a pan-resistant melioidosis, likely associated with the biofilm-forming ability of the bacteria. As in many reported cases, the patient had diabetes, which is regarded as a significant risk factor. ${ }^{14,16}$ Being a farmer with a diabetic foot ulcer, he likely acquired the pathogen through direct contact with contaminated water droplets or soil through a penetrating wound or existing skin abrasion.

The B. pseudomalle $i$ we found in this case were moderate biofilm-formers. Chronic infection and colonization of infective biofilms can occur on dead tissue or medical devices. ${ }^{17}$ Bacterial biofilm can be up to 1,000 times more resistant to antimicrobial agents than their free-living (planktonic) counterpart. ${ }^{18}$ Biofilms, although not correlated with bacterial virulence, ${ }^{21}$ present a barrier, limiting diffusion and eventually the activity of the antibiotics. ${ }^{19,20}$ The full depth of the biofilm is composed of polymeric substances that impede penetration of antimicrobial agent. Moreover, bacterial cells within the biofilm are in a starved state, and are thus slow-growing..$^{18}$ Slow or nongrowing bacterial cells are less or even unsusceptible to antimicrobial agents. ${ }^{22}$ Recent studies found that stimulating B. pseudomallei biofilm-forming ability resulted in upregulation of some genes responsible for increased resistance toward antimicrobial agents. ${ }^{23}$ In addition, biofilm formation of $B$. pseudomallei in vitro is found to be associated with relapse in human melioidosis. ${ }^{8}$

The current recommendation for management of all forms of melioidosis consists of an intensive phase followed by an eradication phase. In the intensive phase, 2 weeks course of ceftazidim or meropenem is prescribed, followed by 12 weeks course of high dose cotrimoxazole. In our case, however, the patient was not given any antibiotics since none were effective. The patient had his foot abscess surgically drained, wound debrided, and total contact casting applied to relieve any pressure off the ulcer. The outcome was good, and the patient was advised to have routine follow-up to monitor the possibility of relapse of infection.
Despite lacking clinical trials, antibiotics alone - with or without incision and drainage - were reported to have successfully treated patients with localized melioidosis, including those with skin and soft tissue lesions and parotid abscesses. ${ }^{13,24-27}$ A case series in Malaysia documented recovery without antibiotics in four children with localized melioidosis after surgical drainage of abscess, and in one case even without surgical treatment. ${ }^{28}$ The current management for biofilm includes sharp debridement; mechanical debridement using curettes, fabric pads, lavage, or ultrasound; and autolytic debridement with moisture-retentive dressings. ${ }^{29,30}$

To the best of our knowledge, this is probably the first report of pan-drug-resistant B. pseudomallei from Java Island, Indonesia. Along with increasing incidence of melioidosis in Indonesia, this report raises the alarm for the possibility of melioidosis in diabetic foot patients in this area. Microbiologists should be aware of the characteristics of the etiologic agent, and cultures should be handled under laboratory Biosafety Level 3 containment. We recommend that all $B$. pseudomallei isolates have their antibiotic sensitivity carefully evaluated.

\section{Ethics statement}

The study was approved by the Medical and Health Research Ethics Committee of the Faculty of Medicine, Public Health and Nursing, Universitas Gadjah Mada, Yogyakarta, Indonesia (KE/FK/0838/EC/2017). Written informed consent was provided by the patient to have the case details and any accompanying images published.

\section{Acknowledgments}

The authors give thanks to Ms Lintang for obtaining patient's data and Mrs Mulyani and Mrs Linda Oktabriana for laboratory assistance.

\section{Disclosure}

The authors report no conflicts of interest in this work.

\section{References}

1. Cheng AC, Currie BJ. Melioidosis: epidemiology, pathophysiology, and management. Clin Microbiol Rev. 2005;18(2):383-416.

2. Redondo MC, Gómez M, Landaeta ME, Eugenia M, R1 H, Khalil R, et al. Melioidosis presenting as sepsis syndrome: a case report. Int J Infect Dis. 2011;15(3):2010-2011.

3. Zueter A, Yean CY, Abumarzouq M, Rahman ZA, Deris ZZ. The epidemiology and clinical spectrum of melioidosis in a teaching hospital in a North-Eastern state of Malaysia: a fifteen- year review. BMC Infect Dis. 2016:1-11.

4. Hemarajata P, Baghdadi JD, Hoffman R, Humphries RM. Burkholderia pseudomallei: Challenges for the Clinical Microbiology Laboratory. $J$ Clin Microbiol. 2016;54(12):2866-2873. 
5. Schweizer HP. Mechanisms of antibiotic resistance in Burkholderia pseudomallei: implications for treatment of melioidosis. Future Microbiol. 2012;7(12):1389-1399.

6. Wuthiekanun V, Amornchai P, Saiprom N, et al. Survey of antimicrobial resistance in clinical Burkholderia pseudomallei isolates over two decades in Northeast Thailand. Antimicrob Agents Chemother. 2011;55(11):5388-5391.

7. Koh SF, Tay ST, Puthucheary SD. Colonial morphotypes and biofilm forming ability of Burkholderia pseudomallei. Trop Biomed. 2013;30(3):428-433.

8. Limmathurotsakul D, Paeyao A, Wongratanacheewin S, et al. Role of Burkholderia pseudomallei biofilm formation and lipopolysaccharide in relapse of melioidosis. Clin Microbiol Infect. 2014;20(11):O854-O856.

9. Dharakul T, Songsivilai S, Viriyachitra S, Luangwedchakarn V, Tassaneetritap B, Chaowagul W. Detection of Burkholderia pseudomallei DNA in patients with septicemic melioidosis. J Clin Microbiol. 1996;34(3):609-614.

10. Dance D. Treatment and prophylaxis of melioidosis. Int J Antimicrob Agents. 2014;43(4):310-318.

11. Clinical and Laboratory Standards Institute. M100 Performance Standards for Antimicrobial Susceptibility Testing. 27th informational supplement; Wayne, PA: Clinical and Laboratory Standards Institute; 2017.

12. Hassan A, Usman J, Kaleem F, Omair M, Khalid A, Iqbal M. Evaluation of different detection methods of biofilm formation in the clinical isolates. Braz J Infect Dis. 2011;15(4):305-311.

13. Gibney KB, Cheng AC, Currie BJ. Cutaneous melioidosis in the tropical top end of Australia: a prospective study and review of the literature. Clin Infect Dis. 2008;47(5):603-609.

14. Currie BJ, Jacups SP, Cheng AC, et al. Melioidosis epidemiology and risk factors from a prospective whole-population study in northern Australia. Trop Med Int Health. 2004;9(11):1167-1174.

15. Kingsley PV, Leader M, Nagodawithana NS, Tipre M, Sathiakumar N. Melioidosis in Malaysia: A Review of Case Reports. PLoS Negl Trop Dis. 2016;10(12):1-18.

16. Benoit TJ, Blaney DD, Gee JE, et al. Melioidosis Cases and Selected Reports of Occupational Exposures to Burkholderia pseudomallei-United States, 2008-2013. MMWR Surveill Summ. 2015;64 (5):1-9.
17. Bryers JD. Medical biofilms. Biotechnol Bioeng. 2008;100(1):1-18.

18. Lewis K. Riddle of biofilm resistance. Antimicrob Agents Chemother. 2001;45(4):999-1007.

19. Pibalpakdee P, Wongratanacheewin S, Taweechaisupapong S, Niumsup PR. Diffusion and activity of antibiotics against Burkholderia pseudomallei biofilms. Int J Antimicrob Agents. 2012;39(4):356-359.

20. Mongkolrob R, Taweechaisupapong S, Tungpradabkul S. Correlation between biofilm production, antibiotic susceptibility and exopolysaccharide composition in Burkholderia pseudomallei bpsI, ppk, and rpoS mutant strains. Microbiol Immunol. 2015;59(11):653-663.

21. Taweechaisupapong S, Kaewpa C, Arunyanart C, et al. Virulence of Burkholderia pseudomallei does not correlate with biofilm formation. Microb Pathog. 2005;39(3):77-85.

22. Panomket P. Burkholderia pseudomallei and biofilms. Asian Biomed. 2015;9(3):285-290.

23. Sawasdidoln C, Taweechaisupapong S, Sermswan RW, Tattawasart U, Tungpradabkul S, Wongratanacheewin S. Growing Burkholderia pseudomallei in biofilm stimulating conditions significantly induces antimicrobial resistance. PLoS One. 2010;5(2):1-10.

24. Achappa B, Madi D, Vidyalakshmi K. Cutaneous melioidosis. J Clin Diagnostic Res. 2016;10(9):WD01-WD02.

25. Ngauy V, Lemeshev Y, Sadkowski L, Crawford G. Cutaneous melioidosis in a man who was taken as a prisoner of war by the Japanese during World War II. J Clin Microbiol. 2005;43(2):970-972.

26. Bodilsen J, Langgaard H, Nielsen HL. Cutaneous melioidosis in a healthy Danish man after travelling to South-East Asia. BMJ Case Rep. 2015;2015:2014-2016.

27. Meckenstock R, Therby A, Marque-Juillet S, et al. Cutaneous melioidosis in adolescent returning from Guadeloupe. Emerg Infect Dis. 2012;18(2):359-360.

28. How HS, Ng KH, Yeo HB, Tee HP, Shah A. Pediatric melioidosis in Pahang, Malaysia. J Microbiol Immunol Infect. 2005;38(5):314-319.

29. Rhoads DD, Wolcott RD, Percival SL. Biofilms in wounds: management strategies. J Wound Care. 2008;17(11):502-508.

30. Metcalf D, Bowler P. Biofilm delays wound healing: A review of the evidence. Burns Trauma. 2013;1(1):5.
International Medical Case Reports Journal

\section{Publish your work in this journal}

The International Medical Case Reports Journal is an international, peer-reviewed open-access journal publishing original case reports from all medical specialties. Previously unpublished medical posters are also accepted relating to any area of clinical or preclinical science. Submissions should not normally exceed 2,000 words or

\section{Dovepress}

4 published pages including figures, diagrams and references. The manuscript management system is completely online and includes a very quick and fair peer-review system, which is all easy to use. Visit http://www.dovepress.com/testimonials.php to read real quotes from published authors. 